\title{
Correction to: Highly efficient, recyclable cerium-phosphate solid acid catalysts for the synthesis of tetrahydrocarbazole derivatives by Borsche-Drechsel cyclization
}

\author{
Hatem M. Altass ${ }^{1,2} \cdot$ Abdelrahman S. Khder $^{1,2,3}$ (D) Saleh A. Ahmed ${ }^{1,2,4}$. \\ Moataz Morad ${ }^{1,2} \cdot$ Abdullah A. Alsabei $^{1,2} \cdot$ Rabab S. Jassas $^{5}$. \\ Khalid Althagafy ${ }^{2,6} \cdot$ Awad I. Ahmed $^{3} \cdot$ Reda S. Salama $^{7}$
}

Published online: 25 October 2021

(c) Akadémiai Kiadó, Budapest, Hungary 2021

\section{Correction to: Reaction Kinetics, Mechanisms and Catalysis (2021) 134:143-161 \\ https://doi.org/10.1007/s11144-021-02050-4}

In the original publication of the article, in the Acknowledgements section, the Project Number was incorrectly given. This has been corrected with this Correction.

Acknowledgements The authors are highly indebted to the Deanship of the Scientific Research (DSR), Umm Al-Qura University for the financial support through the Project Number 19-SCI-1-01-0049.

The original article can be found online at https://doi.org/10.1007/s11144-021-02050-4.

Abdelrahman S. Khder

askhder@uqu.edu.sa

1 Chemistry Department, Faculty of Applied Science, Umm Al-Qura University, Makkah 21955, Saudi Arabia

2 Research Laboratories Unit, Faculty of Applied Science, Umm Al-Qura University, Makkah 21955, Saudi Arabia

3 Faculty of Science, Chemistry Department, Mansoura University, Mansoura 35516, Egypt

4 Chemistry Department, Faculty of Science, Assiut University, Assiut 71516, Egypt

5 Department of Chemistry, Jamoum University College, Umm Al-Qura University, Makkah 21955, Saudi Arabia

6 Physics Department, Faculty of Applied Science, Umm Al-Qura University, Makkah 21955, Saudi Arabia

7 Basic Science Department, Faculty of Engineering, Delta University for Science and Technology, Gamasa, Egypt 
Publisher's Note Springer Nature remains neutral with regard to jurisdictional claims in published maps and institutional affiliations. 